\title{
Die Bewertung von Genussscheinen zwischen Fremd- und Eigenkapitalcharakteristika1
}
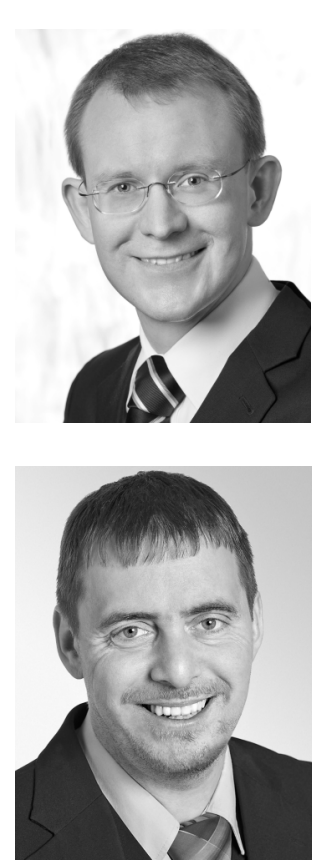

\author{
J. Henning Fock, Andreas W. Rathgeber
}

Mezzanines Kapital, Genussschein, Strukturelles Modell, EUWAX

Mezzanine Capital, Profit Participation Certificates, Structural Model, EUWAX

Durch die Finanzkrise wurde der mezzanine Charakter von Genussscheinen evident, da Verluste der Banken ohne Auftreten des Insolvenztatbestandes zur Aussetzung von Ausschüttungen und zur Verminderung von Rückzahlungsansprüchen der Genussscheine führten. Mit dem hier analog zu US-Vorzugsaktien entwickelten Genussscheinmodell können, anders als mit gebräuchlichen Modellen zur Bewertung von Fremdkapital, die mezzaninen Eigenschaften der Genussscheine berücksichtigt werden. Das Bewertungsmodell wurde empirisch anhand von 3.300 Kurstagen für 130 an der EUWAX gehandelte Genussscheine überprüft. Hierbei wurde die Anpassungsgüte des Genussscheinmodells derjenigen des Merton- und des Kanders-Modells gegenübergestellt. Als zentrales Ergebnis zeigte sich eine mindestens gleich gute Anpassbarkeit des Genussscheinmodells an Marktpreise in der Normalsituation. Eine bessere Anpassbarkeit konnte beobachtet werden, wenn der mezzanine Charakter der Genussscheine zum Tragen kam, was bei gleichzeitigem Vorliegen von mehr als drei umsatztragenden Kursen fast immer gegeben war. Insgesamt zeigt die empirische Untersuchung, dass mit dem neuen Modell eine wesentliche Verbesserung gegenüber den bestehenden Modellen erreicht wird. Die Teilnehmer des Genussscheinmarkts scheinen in die Bewertung die Genussscheincharakteristika miteinzubeziehen, obwohl gerade diese Charakteristika Aussenstehenden oft nicht oder nicht in vollem Umfang bekannt sind und der Markt dadurch undurchsichtig wirkt.

The mezzanine character of profit participation certificates, a category of securities known in Germany as "Genussscheine" (GS), became evident during the financial crisis as banks' losses lead to the suspension of dividends and to the reduction of redemption claims without the banks going bankrupt. With the new GS model which is build analogous to models for the pricing of US preferred stocks the mezzanine characteristics of GS can be taken

1 Die Autoren bedanken sich erstens bei der Stuttgarter Börse (EUWAX), die dieses Projekt mit Daten unterstützt hat; zweitens für wertvolle Hinweise bei den Teilnehmern der folgenden Tagungen: 22. Jahreskonferenz der Multinational Finance Society 2015, Kölner Finanzmarktkolloquium 2007, Campus for Finance Conference 2007 und Jahrestagung der Schweizerische Gesellschaft für Finanzmarktforschung 2006. Drittens geht unser Dank namentlich an Günter Bamberg, Wolfgang Breuer, Tobias Gaugler, Leonhardt Grünwald, Manfred Steiner, Martin Wallmeier und an den anonymen Gutachter für wertvolle Anregungen. 
into account which is not possible with the existing models for the pricing of debt capital. An empirical evaluation with 3300 prices of 130 GS traded at the EUWAX exchange was performed and we compare the adjustment quality of the new GS model to the one of the Merton and Kanders model. Out-of-sample the GS model often delivers better results than existing models which is especially the case if the mezzanine character of GS came into play which is mostly the case if more than 3 GS prices were available. Altogether the empirical evaluation shows that with the new GS model a substantial improvement can be achieved and that on the opaque GS market the participants take the specific characteristics of GS into account for pricing.

\section{Einleitung}

Denken Investoren an Genussscheine, ${ }^{2}$ denken sie häufig an sehr spezielle Finanzinstrumente, deren Zahlungen von Dividenden, Gesamtkapital- oder Umsatzrenditen der begebenden Unternehmen abhängen (siehe etwa Kanders 1990 oder Schäcker 1997). Dabei ist das Gros der Genussscheine sehr ähnlich und deutlich einfacher strukturiert und dadurch mit festverzinslichen Wertpapieren vergleichbar (Lühn 2013, S. 68 f. oder Brokamp et al. 2008, S. 114), weswegen institutionelle und private Anleger diese als interessante Anlagealternative zu festverzinslichen Wertpapieren ausgemacht haben (Kühn 2008, S. 53). Allerdings bewegt sich die Bedeutung des Genussscheinmarktes in Zyklen. So stieg die Anzahl der notierten Genussscheine von 43 Genussscheinen im Jahr 1990 auf 112 im Jahr 2009, um dann bis ins Jahr 2015 auf unter 50 Genussscheine abzunehmen. (EUWAX und Lühn 2006, S. 10 ff.). ${ }^{3}$ Der Fokus liegt hierbei auf dem deutschen Markt, da sich der Genussschein als Möglichkeit der stimmrechtsmehrheitserhaltenden Kapitalaufnahme nur mit Modifikationen in wenigen anderen Ländern, insbesondere im deutschsprachigen Raum, etabliert hat (Ernst 1963, S. 43 ff., Lühn 2006, S. 285).

Allerdings unterscheiden sich festverzinsliche Wertpapiere, auch nachrangige Papiere, und Genussscheine fundamental. Denn im Gegensatz zu den meisten nachrangigen Verbindlichkeiten, die nur im Insolvenzfall nicht bedient werden, kann es bei Genussscheinen auch ohne Insolvenz des Emittenten zum (Teil-)Verlust des eingesetzten Kapitals kommen (Lühn 2013, S. 48). Bei Genussscheinemissionen durch Kreditinstitute ist dies zwingend vorgeschrieben, damit die meist von Emittentenseite angestrebte Anrechnung als Ergänzungskapital möglich wird. ${ }^{4}$ Verluste führen hierbei zu ausgefallenen Zinszahlungen und reduzieren im Extremfall sogar den Rückzahlungsanspruch der Genussscheininhaber am Laufzeitende.

Dieses Charakteristikum dokumentiert exemplarisch die Stellung von Genussscheinen zwischen Eigen- und Fremdkapital. Insgesamt führen diese Regelungen dazu, dass in Zeiten, in denen es den Emittenten wirtschaftlich gut geht, der Fremdkapitalcharakter der Genussscheine dominiert und Genussscheine aufgrund der vereinbarten Verzinsung ähnlich wie nachrangige festverzinsliche Anleihen bewertet werden (Westphal 1995, S. $23 \mathrm{ff}$. oder S. 175 ff.). Damit eröffnet sich auch die Möglichkeit der Bewertung dieser Instrumente über z.B. auf Bonitätsrisikoprämien kalibrierte Reduktions- oder auch strukturelle Model-

2 Die rechtliche Ausgestaltung des dt. Genussscheins entspricht in schweizerischem und österreichischem Recht der des Partizipationsscheins.

3 Ähnliches gilt für das Marktvolumen, dass sich in der Spitze im Jahr 2009 bei 17 Mrd € bewegte.

4 Allerdings sieht der neueste Stand des KWG vor, nicht mehr Genussscheinkapital und nachrangige Verbindlichkeiten zu unterscheiden (siehe auch Fn. 3). 
le (Hirte 2001, Sp. 973 oder Götte 2007, S. 22 ff.). In Zeiten der Finanzkrise ist diese Sichtweise nicht mehr zielführend, da aufgrund zahlreicher realisierter oder drohender Bilanzverluste die Rückzahlungsansprüche aufgrund der Verlustteilnahme nun unter dem Nennwert der jeweiligen Papiere liegen (Rottwilm 2010 oder Lühn 2012).

Um dieser Dualität, den Fremd- und Eigenkapitalaspekten, Genüge zu tun, benötigt man ein Bewertungsmodell, das beide gleichzeitig berücksichtigt. Zwar existieren in der Literatur zahlreiche Modelle, die den einen oder den anderen Aspekt betonen (vgl. etwa Nowak/Hartman 1995, S. 414 f.) und sich auch intensiv mit Spezialfällen auseinandersetzen (vgl. etwa Kanders 1990, S. 97); eine Betrachtung des oben beschriebenen Standardfalles, der für mehr als 80\% der börsengehandelten Genussscheine (vgl. hierzu Abschnitt 2.1) zutrifft, scheint nach Recherche der Autoren zu fehlen. Hieraus ergibt sich die Motivation dieses Beitrags, eben ein solches Modell zu entwickeln.

Um diese Lücke zu schliessen, bedient sich dieser Beitrag der Familie der strukturellen Modelle, deren generelles Kennzeichen u.a. die Modellierung des Unternehmensvermögens als stochastischer Prozess ist. Damit steht eine Methode zur Verfügung, die bei genussscheinähnlichen Finanzprodukten, wie amerikanischen Vorzugsaktien, bereits erfolgreich eingesetzt wurde. So bewertet Emanuel (1983) als einer der ersten nicht in Stammaktien umwandelbare US-amerikanische Vorzugsaktien mit unendlicher Laufzeit, die mit einer fixen Dividende ausgestattet sind, mit einem strukturellen Modell. Der grundsätzliche Aufbau dieses Modells kann auf Genussscheine übertragen werden. Die unendliche Laufzeit sowie der Ausschluss der Insolvenz schliessen jedoch einen isomorphen Transfer aus, so dass sich auch die empirischen Ergebnisse bzgl. der Vorzugsaktien nicht auf deutsche Genussscheine übertragen lassen. Für Genussscheine stellt sich also die Frage, inwieweit ein an den amerikanischen Vorzugsaktien orientiertes Bewertungsmodell - insbesondere im Hinblick auf die jüngsten Entwicklungen an den Finanzmärkten - bessere Ergebnisse erzielen kann als die bisher vorhandenen Modelle.

Ziel dieses Beitrags ist es, ein Modell zu entwickeln, mit dem die Bewertung der überwiegenden Mehrheit der am Sekundärmarkt notierten Genussscheine aus Investorensicht erfolgen kann und das deren Stellung zwischen Fremd- und Eigenkapital abbildet, was das explizite Miteinbeziehen von spezifischen Merkmalen, wie Ausfall der Zinszahlung im Verlustfall, Verlustteilnahme oder begrenzte Laufzeit, impliziert.

Dazu werden im nachfolgenden Abschnitt die typischen Eigenschaften der Mehrzahl der Genussscheine beschrieben und formalisiert. Aufbauend auf dem typischen Annahmebündel der Bewertungsmodelle von Vorzugsaktien werden dann ein risikoneutralisierter Preisprozess wie auch theoretische Preise für Genussscheine abgeleitet. Um die Eignung des Modells zu überprüfen, werden im letzten Abschnitt die Modellpreise mit Marktpreisen von 130 Genussscheinen aus den Jahren 2002 bis 2009 verglichen. Als Referenzwerte für einen In-Sample und Out-of-Sample Test dienen das Merton-Modell (Merton 1974) als Kreditrisikomodell und das Kanders-Modell (Kanders 1990) als Modell für spezielle eigenkapitalähnliche Genussscheine. Die Ergebnisse werden in der abschliessenden Zusammenfassung im Vergleich zu den bereits bestehenden Modelle diskutiert, um in letzter Konsequenz einen Vorschlag zu erarbeiten, wie die weit über 1000 von Industrieunternehmen begebenen und nicht börsennotierten mezzaninen Finanzinstrumente bewertet werden können. 


\section{Definitionen und Annahmebündel}

\subsection{Institutionelle Gegebenheiten}

Die Eigenschaften von Genussscheinen sind in Deutschland nicht durch Gesetze einheitlich vorgegeben. Die einzige allgemeine Erwähnung findet sich in $\$ 221$ (3) und (4) Aktiengesetz (AktG), in dem die Ausgabe von Genussrechten an Bedingungen, wie einen Hauptversammlungsbeschluss, geknüpft wird (Brokamp et al. 2008, S. 115). Deswegen basieren Rechte und Pflichten der Genussscheine auf einer einzelvertraglichen Regelung zwischen Emittent und Investor, die im jeweiligen Prospekt kodifiziert ist. Grundsätzlich sind damit Genussscheine, was die Zahlungsstruktur, bestehend aus Kuponzahlungen, Rückzahlung des Nominalbetrages und Fristigkeit betrifft, individuell vereinbar. Sie können somit verschiedenste Formen, wie etwa gewinnabhängige Ausschüttungen oder keine Laufzeitbegrenzung, annehmen.

Es existiert jedoch eine grosse Gruppe von Genussscheinen mit ähnlichen Charakteristi$\mathrm{ka}$, da diese von Kreditinstituten emittiert wurden, um als Ergänzungskapital nach $\mathbb{} 10$ (5) KWG (4. bis 7. Novelle $)^{5}$ herangezogen werden zu können. Im weiteren Verlauf wird genau dieser KWG-konforme Typ von Genussscheinen, der sich auch auf Genussscheine anderer Emittenten ausgewirkt hat, betrachtet.

Diesen KWG-konformen Standardtyp von Genussscheinen kennzeichnet eine Übereinstimmung in den drei typischen finanzwirtschaftlichen Grössen eines Anleihezahlungsstroms, also der Zinszahlung, der Rückzahlung sowie den Zahlungsterminen (siehe auch ähnlich das Modell Typ A bei Kanders 1990, S. 50 i.V.m. S. 136).

1. Die Zinszahlung (Kupon) erfolgt dabei nur solange, bis durch diese nach $\mathbb{1 0}$ (5) Nr. 1 KWG kein Verlust, in der Interpretation der meisten Genussscheinbedingungen, kein Bilanzverlust oder ein Jahresfehlbetrag (Lühn 2013, S. 50), entsteht. Ergänzend wird stets ein prioritätischer Gewinnanteil gegenüber den Eigenkapitalgebern vereinbart. (Westphal 1995, S. 105).

2. Im Falle eines Verlusts verlangt $\mathbb{1 0}$ (5) Nr. 1 KWG eine Teilnahme an diesem durch die Reduktion des Rückzahlungsanspruchs der Genussscheine. Diese Reduktion erfolgt partizipativ, d.h., mit derselben Quote, mit der auch die Eigenkapitalgeber am Verlust teilnehmen, wird der Rückzahlungsanspruch reduziert. Als Basis für diese Berechnung wird hier das Eigenkapital zu Beginn des Geschäftsjahres, meist das bilanzielle Eigenkapital, teilweise das Kernkapital inklusive der Vorsorgereserven nach $\mathbb{3} 340 \mathrm{~g}$ HGB, verwendet.

3. Ausgefallene Zinszahlungen können in späteren Jahren nur im Nichtverlustfall (zum Verlust: siehe Punkt 1) nachgezahlt werden, wobei diese Nachzahlungen als stets prioritär angesehen werden können. ${ }^{6}$

5 Im Folgenden wird auf die 4. bis 7. Novellen des KWG abgestellt, da diese die Emissionsbedingungen eines Grossteils der Genussscheine im Datensatz determiniert haben und im Gegensatz zur Situation nach den Änderungen des KWG im Jahr 2014 explizite Forderungen an Emissionsbedingungen von Genussscheinen aufweisen. Letztendlich haben die Regelungen des KWG bis 2014 auch eine Ausstrahlung auf neuere Emissionen.

6 Die einzige Ausnahme bildet die gesetzliche Rücklage nach $\mathbb{S} 150$ (2) AktG, die bei Aktienbanken vorrangig aus dem Jahresüberschuss bedient werden muss. Da es sich bei den meisten Genussscheinemittenten um Kreditinstitute handelt, deren Rücklagen bei Weitem die gesetzlich geforderte Mindestrücklage von $10 \%$ (in der Stichprobe durchschnittlich 500\%) überschreiten, greift diese Vorabzuführung zu den Rücklagen in der Praxis der Genussscheinemittenten nicht. An dieser Tatsache ändert auch ein Jah- 
4. Eine Reduktion des Rückzahlungsanspruchs kann durch nachfolgende Gewinne vermindert werden. Dabei wird die Wiederauffüllung des Rückzahlungsanspruchs stets vorrangig zu den ausgefallenen Zinszahlungen behandelt, erfolgt jedoch de facto partizipativ (zur partizipativen Beteiligung: siehe Punkt 2) (Westphal 1995, S. 121). ${ }^{7}$

5. Massgeblich für die Zinszahlung ist die Feststellung bzw. Billigung des Jahresabschlusses durch Haupt-, Mitglieder- oder Trägerversammlung, sodass die Zahlungstermine durch diese determiniert werden (siehe auch Abbildung 1). Dies impliziert erstens, dass das Ende des Geschäftsjahres, in dem ein für die Höhe der Kuponzahlung massgeblicher Gewinn erwirtschaftet wird, deutlich vor dem Zahlungstermin liegt. Zweitens weicht auch der nominelle Endfälligkeitszeitpunkt von dem der Rückzahlung ab, was durch ein Versprechen einer zusätzlichen unterjährig linear verzinslichen Kuponzahlung für diesen Zeitraum abgegolten wird. Für die zusätzliche Zahlung ist ausreichend, dass durch die Kuponzahlung für das letzte Geschäftsjahr kein Verlust entsteht.

Einschränkend muss noch erwähnt werden: Die Möglichkeit einer Nachzahlung von Zinsen oder die Erhöhung des reduzierten Rückzahlungsanspruchs ist bei allen Genussscheinen nur während der Laufzeit nach Auslegung des $\mathbb{1 0}(5) \mathrm{Nr} .5 \mathrm{KWG}$ möglich.

Zur Bestimmung des Zahlungsstroms ist darüber hinaus die Möglichkeit einer Insolvenz mit zu berücksichtigen. Grundsätzlich besteht hier fast immer die Regelung, dass bei einer Insolvenz die Genussscheininhaber bevorzugt oder zumindest gleichrangig wie das übrige Eigenkapital beteiligt werden (Lühn 2013, S. 48). ${ }^{8}$

Auch wenn dies nun als sehr spezieller Genussscheintyp angesehen werden könnte, bleibt festzuhalten, dass dieser Typ der Genussscheine mit fast 80\% Marktanteil den bei weitem größten Anteil unter den Genussscheinen darstellt, die an der EUWAX notiert sind. ${ }^{9}$ Lühn (2006, S. 11) kommt zu ähnlichen Ergebnissen für frühere Jahre und Westphal (1995, S. 164) zeigt, dass in den 1980er Jahren eine Entwicklung von unstandardisierten Genussscheintypen zu dem KWG konformen Standardtyp stattgefunden hat. Darüber hinaus sind 98\% der Genussscheine an der EUWAX mit einem fixen oder von einem Refe-

resfehlbetrag nichts, da letzterer zu keiner Rücklagenreduktion, sondern nach den Ausführungen der Fn. 5 zu einem Verlustvortrag führt.

7 Diese Abweichung von der mit Ausnahme der Bildung der gesetzlichen Rücklage vorrangingen Wiederauffüllung des Rückzahlungsanspruchs entsteht wie folgt: Die gängige Praxis ist nämlich, im Verlustfall einen Verlustvortrag im Sinne des $\$ 268$ (1) HGB zu bilden. (vgl. DGAP 2010a oder DGAP 2010b). Bei Gewinnen in den Folgejahren werden diese zum einen Teil zur Wiederauffüllung der Rückzahlungsansprüche der Genussrechtsinhaber und zum anderen Teil zur Verminderung des Verlustvortrags verwendet. Eine sofortige Verrechnung des Jahresfehlbetrags mit den Rücklagen schliesst die Möglichkeit der anteilsgemässen Wiederauffüllung aus, wird aber von den leitungsbefugten Eigenkapitalgebern in praxi nicht bevorzugt werden, womit de facto eine partizipative Wiederauffüllung besteht. Hierzu ist noch anzumerken, dass bei Verwendung des Bilanzverlustes als Bemessungsgrundlage, im Falle eines Verlustes des Vorjahres, der Bilanzgewinn im nächsten Jahr wiederum vermindert wird. Somit kann der Genussscheininhaber unter Umständen an einem Jahresfehlbetrag mehrere Jahre negativ partizipieren. Ein Beispiel für die Praxis bei der Emission dieser Variante sind einige Genussscheine der IKB (vgl. DGAP 2010b). Bei vielen börsennotierten Genussscheinen wird die Möglichkeit der Mehrfachteilnahme am Verlust aber explizit im Prospekt ausgeschlossen.

8 Für das Eintreten der Insolvenz gelten die üblichen Bestimmungen: Zahlungsunfähigkeit gemäss $\$ 17$ InsO oder Überschuldung $\mathbb{S} 19$ InsO. Ferner ist für Kreditinstitute als Emittenten zu berücksichtigen, dass aufgrund aufsichtsrechtlicher Bestimmungen ( $\$ 45 \mathrm{KWG}$ ) im Falle der drohenden Überschuldung Zwangsmassnahmen wie eine Restrukturierung eingeleitet werden würden.

9 Von den im Dezember 2009 notierten 112 Genussscheinen an der EUWAX waren nur 24 nicht KWGkonform. Quelle EUWAX. 
renzzins abhängigen Zins ausgestattet. ${ }^{10} \operatorname{Lühn}$ (2006, S. 15) kommt für frühere Jahre zu ähnlichen Ergebnissen. Zu leicht niedrigeren Ergebnissen kommt Kanders (1990, S. 50) für die 1980er Jahre. Flankiert wird diese Entwicklung hin zur Standardisierung durch eine grössere Umschlaghäufigkeit der Genussscheine (Steinbach 1999, S. 151).

\subsection{Aufbau des Zahlungsstroms}

Im Folgenden wird ausschliesslich der in 2.1 beschriebene KWG-konforme GenussscheinTyp behandelt. Dessen rechtliche Ausgestaltung wird im Folgenden in eine finanzwirtschaftliche Modellform gegossen. Um Punkt 5 aus 2.1 Genüge zu tun, werden zur Modellierung des Zahlungsstroms und der zugehörigen Geschäftsjahre zwei Folgen von Zeitpunkten benötigt, wobei $\theta_{\mathrm{k}}(\mathrm{k} \in(1,2 . . \mathrm{K}))$ die Zahlungstermine und $\mathrm{t}_{\mathrm{i}}(\mathrm{i} \in(1,2 . .1))$ die Enden der Geschäftsjahre bezeichnet. $\Theta_{\mathrm{k}}$ steht ferner für die Zeiträume vom Bewertungszeitpunkt $\mathrm{t}$ bis zur Fälligkeit der einzelnen Zahlungen $\theta_{\mathrm{k}}, \mathrm{T}_{\mathrm{i}}$ für den Zeitraum vom Bewertungszeitpunkt $\mathrm{t}$ bis zum Geschäftsjahresende $\mathrm{t}_{\mathrm{i}}$ (siehe Abbildung 1). An diesem Rückzahlungstermin fliesst neben dem Kupon $\mathrm{C}$ und dem Rückzahlungsbetrag R des Genussscheins, beides in \% des Nominalbetrags gemessen, dem Investor die erwähnte zusätzliche Kuponzahlung $C\left(\Theta_{K}-T_{I}\right) \mathrm{zu}$.

Zur Bestimmung der Zahlungshöhe wird an den Enden der Geschäftsjahre der Bilanzgewinn oder ein Jahresüberschuss $\Delta \mathrm{V}_{\mathrm{i}}$ festgestellt. Eine Teilnahme am Verlust wird durch die Reduktion des Rückzahlungsanspruchs um $\mathrm{VB}_{\mathrm{k}}$ in \% des Nominalbetrags gemessen. Dabei wird nur ein Teil $\alpha^{11}$ des Verlusts durch die Genussscheininhaber mit Genussrechtskapital $\mathrm{GK}_{\mathrm{i}-1}$ getragen, wobei $1-\alpha$ die Eigenkapitalgeber $\mathrm{EK}_{\mathrm{i}-1}$ zu Beginn des Geschäftsjahres übernehmen. Beim Ausfall der Kuponzahlung erhält der Investor einen Nachzahlungsanspruch in Höhe von $\mathrm{N}_{\mathrm{k}}$ in den späteren Geschäftsjahren, der wie die Reduktion der Verlustbeteiligung mit dem Ende der Laufzeit erlischt.

10 Zwar wird im Schrifttum teilweise angezweifelt (Gehling 1992 oder Ernst 1963, S. 110 ff.), dass es sich hierbei um Genussscheine handelt, doch der Meinung, dass hier Obligationen vorliegen, kann nicht gefolgt werden (Eberhartinger 1996, S. 14).

$11 \alpha$ wird dabei als annähernd konstant angenommen, obwohl es im Modell aufgrund von Einzahlungen für ausgeschüttete Dividenden oder Einzahlungen für Zahlungen für Genussscheininhaber leicht schwankt. Diese Vorgehensweise entspricht auch teilweise den Genussscheinprospekten, bei denen implizit der Parameter fix vorgegeben ist. 


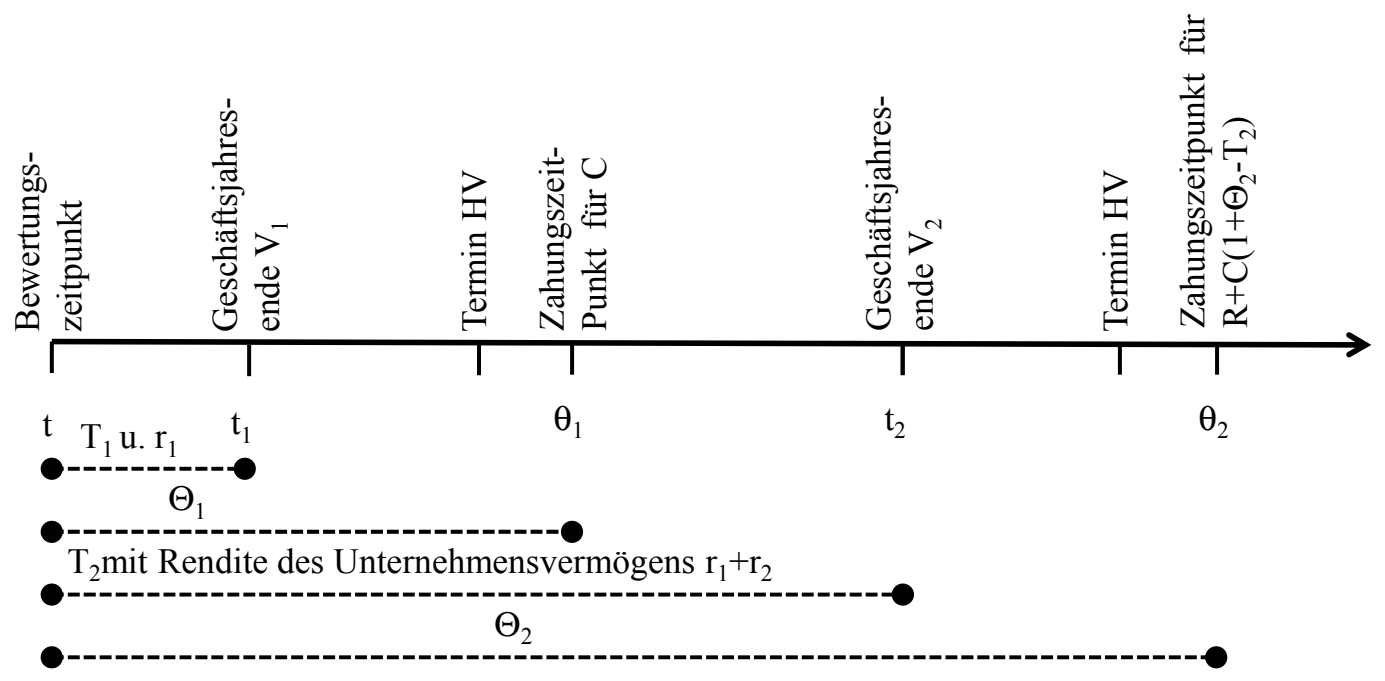

Abbildung 1: Zahlungszeitpunkte und Geschäftsjahresende eines zweijährigen Genussscheins

Hierbei sind drei Komponenten für Auszahlung bzw. Nachzahlungsanspruch während der Laufzeit zu unterscheiden:

a) Für die Zahlung (Punkt 1 aus 2.1) wird, falls der Gewinn ${ }^{12} \Delta \mathrm{V}_{\mathrm{i}}$ am Ende des Geschäftsjahres i nicht für die Bedienung von Kupon- $C$ und evtl. Nachzahlungen $\mathrm{N}_{\mathrm{k}}$ zum Zahlungszeitpunkt $\mathrm{k}$ ausreicht, nur der Gewinn des Emittenten in Relation zum Genussrechtskapital gezahlt (Teilzahlung). Im Falle eines ausreichenden Gewinns wird, abhängig vom Vorjahr, entweder der Kupon oder der Kupon zuzüglich eines Nachzahlungsanspruchs gezahlt (Vollzahlung).

b) Der Nachzahlungsanspruch $\mathrm{N}_{\mathrm{k}}$ (Punkt 3 aus 2.1) umfasst im Verlustfall in Abhängigkeit vom Vorjahr entweder den Kupon C oder den Kupon zuzüglich weiterer Nachzahlungsansprüche aus den Vorjahren. Im Falle eines nicht ausreichenden Gewinns wird dieser Betrag um den ausgeschütteten Gewinnanteil, den Gewinn in Relation zum Genussrechtskapital, vermindert. Der Nachzahlungsanspruch $\mathrm{N}_{\mathrm{k}}$ über mehrere Perioden setzt sich aus der Summe der einzelnen Nachzahlungen zusammen. Er ist dadurch eine kumulierte Grösse und pfadabhängig.

c) Bei der Verlustbeteiligung $\mathrm{VB}_{\mathrm{k}}$ (Punkt 2 aus 2.1) erfolgt keine Auszahlung. Hier wird anteilig der Verlust zur Minderung des Rückzahlungsanspruchs $\mathrm{R}$ verwendet. Im Falle eines Verlusts im Vorjahr kumuliert sich die Verlustbeteiligung.

Insgesamt lassen sich für jede der Komponenten drei mögliche Fälle unter Berücksichtigung eventueller Verlustbeteiligungen aus der Vergangenheit, Gewinn $\geq$ Kupon- + Nachzahlungen, Gewinn < Kupon- + Nachzahlungen und Verlust, für den aktuellen Zahlungszeitpunkt in Kombination mit drei möglichen Fällen für das Vorjahr während der Laufzeit

12 Aus Vereinfachungsgründen wurde hier die Bezeichnung Gewinn gewählt. Dazu ist stets zu berücksichtigen, dass der Gewinn abzüglich einer noch bestehen Verlustbeteiligung (anteilig für Eigen- und Genussrechtskapitalgeber) gemeint ist. In den Berechnungen wurde dies dagegen stets berücksichtigt. 
der Genussscheine ableiten, wie Tabelle 1 zeigt. Als Notation verwenden wir für die Zahlung $\mathrm{CF}_{\mathrm{k}}$.

\begin{tabular}{|c|c|c|c|c|}
\hline Variable & $\begin{array}{l}\text { Aktuelles Jahr } k \\
\text { Vorjahr } k-1\end{array}$ & $\begin{array}{c}\text { Vollzahlung: } \\
\text { Gewinn ant. VB } \geq \\
\text { Kupon- + Nachzahl. }\end{array}$ & $\begin{array}{c}\text { Teilzahlung } \\
\text { Gewinn - ant. VB }<\text { Kupon- + } \\
\text { Nachzahlungen }\end{array}$ & $\begin{array}{l}\text { Keine Zahlung } \\
\text { Verlust }\end{array}$ \\
\hline \multirow{3}{*}{$\begin{array}{l}\text { Zahlung } \\
\mathrm{CF}_{\mathrm{k}}\end{array}$} & $\begin{array}{l}\text { Gewinn - ant. VB } \geq \\
\text { Kupon- + Nachzahl. } \\
\mathrm{N}_{\mathrm{k}-1}\end{array}$ & $C$ & $\frac{\Delta V_{i}}{\mathrm{GK}_{i}}$ & 0 \\
\hline & $\begin{array}{l}\text { Gewinn - ant. VB< } \\
\text { Kupon- + Nachzahl. } \\
\text { N }_{\text {k-1 }}\end{array}$ & $C+N_{k-1}$ & $\frac{\Delta V_{i}}{\mathrm{GK}_{i}}$ & 0 \\
\hline & Verlust $\mathrm{VB}_{\mathrm{k}-1}$ zzgl. $\mathrm{N}_{\mathrm{k}-1}$ & $C+N_{k-1}$ & $\frac{\Delta V_{i}}{\mathrm{GK}_{i}}+\frac{\mathrm{VB}_{k-1}}{\alpha}$ & 0 \\
\hline \multirow{3}{*}{$\begin{array}{l}\text { Nachzah- } \\
\text { lungsan- } \\
\text { spruch } \mathrm{N}_{\mathrm{k}}\end{array}$} & $\begin{array}{l}\text { Gewinn - ant. VB } \geq \\
\text { Kupon- + Nachzahl. } \\
\mathrm{N}_{\mathrm{k}-1}\end{array}$ & 0 & $C-\frac{\Delta V_{i}}{\mathrm{GK}_{i}}$ & C \\
\hline & $\begin{array}{l}\text { Gewinn - ant. VB }< \\
\text { Kupon- + Nachzahl. } \\
\mathrm{N}_{\mathrm{k}-1}\end{array}$ & 0 & $C-\frac{\Delta V_{i}}{\mathrm{GK}_{i}}+N_{k-1}$ & $C+N_{k-1}$ \\
\hline & Verlust $\mathrm{VB}_{\mathrm{k}-1}$ zzgl. $\mathrm{N}_{\mathrm{k}-1}$ & 0 & $C-\frac{\Delta V_{i}}{\mathrm{GK}_{i}}+N_{k-1}+\frac{\mathrm{VB}_{k-1}}{\alpha}$ & $C+N_{k-1}$ \\
\hline \multirow{3}{*}{$\begin{array}{l}\text { Verlustbe- } \\
\text { teiligung } \\
\mathrm{VB}_{\mathrm{k}}\end{array}$} & $\begin{array}{l}\text { Gewinn - ant. VB } \geq \\
\text { Kupon- + Nachzahl } \\
\mathrm{N}_{\mathrm{k}-1}\end{array}$ & 0 & 0 & $\alpha \cdot \frac{\Delta V_{i}}{\mathrm{GK}_{i}}$ \\
\hline & $\begin{array}{l}\text { Gewinn - ant. VB < } \\
\text { Kupon- + Nachzahl. } \\
\mathrm{N}_{\mathrm{k}-1}\end{array}$ & 0 & 0 & $\alpha \cdot \frac{\Delta V_{i}}{\mathrm{GK}_{i}}$ \\
\hline & Verlust $\mathrm{VB}_{\mathrm{k}-1}$ zzgl. $\mathrm{N}_{\mathrm{k}-1}$ & 0 & 0 & $\alpha \cdot \frac{\Delta V_{i}}{\mathrm{GK}_{i}}+\mathrm{VB}_{k-1}$ \\
\hline
\end{tabular}

Tabelle 1: Zahlungen und Nachzahlungsanspruch für eine Einheit Nominalbetrag mindestens ein Jahr vor dem Fälligkeitszeitpunkt

Am Ende der Laufzeit bestehen keinerlei Nachzahlungsansprüche oder Möglichkeiten mehr, die Verlustbeteiligung zu reduzieren. Im für den Investor besten Fall wird der gesamte Kupon $C\left(1+\Theta_{K}-T_{I}\right)+\mathrm{R}$ zzgl. eventueller Nachzahlungen $\mathrm{N}_{\mathrm{K}-1}$ und der Rückzahlungsbetrag ausbezahlt. Im Falle, dass der Gewinn nicht dazu ausreicht, wird neben der Rückzahlung der gesamte Gewinn prioritär an die Genussscheininhaber ausgeschüttet $\frac{\Delta \mathrm{V}_{I}}{\mathrm{GK}_{I}}+R$. Die Zahlungen an Genussscheingläubiger sind bevorrechtigt vor den Eigenkapitalgebern. Im Verlustfall wird der Rückzahlungsbetrag um die Verlustbeteiligung für das aktuelle und für evtl. in früheren Jahren angefallene Verluste partizipativ gekürzt $R+\alpha \cdot \frac{\Delta \mathrm{V}_{I}}{\mathrm{GK}_{I}}+\mathrm{VB}_{K-1}$. Die Nachzahlungen summieren sich über die Laufzeit auf und sind damit in ihrer Höhe pfadabhängig.

Ferner wird im Folgenden angenommen, dass im Insolvenzfall der Genussscheininhaber keine Zahlungen mehr erhält. Aus Vereinfachungsgründen wird für den Insolvenzfall nur auf den Tatbestand der Überschuldung referenziert. Dieser Fall tritt im Modell annahmegemäss dann ein, wenn das bilanzielle Eigenkapital aufgebraucht ist. Genussscheine wirken damit nicht insolvenzauslösend. Wenn für den Gewinn

$\Delta V_{i}<-\mathrm{EK}_{i}-\mathrm{GK}_{i}-\mathrm{VB}_{i-1} \cdot \mathrm{GK}_{i-1}$ 
gilt, ist der Emittent insolvent. Dies ist gleichbedeutend mit der Tatsache, dass das Vermögen unter den fixen Fremdkapitalbestand $L$ (Konsol) fällt: $L>V_{i}$. Zur Vereinfachung der Modellierung wird hierbei angenommen, dass eine Insolvenz nur zum Zeitpunkt des jeweiligen Bilanzstichtages eintreten kann.

\subsection{Annahmen für das Vermögenswertmodell (insb. den stochastischen Prozess)}

In den skizzierten Eigenschaften ähneln damit Genussscheine amerikanischen Vorzugsaktien. Nahe liegend ist es daher, bei der Bewertung auf die Modellfamilie für amerikanische Vorzugsaktien etwa von Emanuel (1983) oder Ferreira et al. (1992) aufzubauen, wobei das Modell jedoch an die Gegebenheiten der Genussscheine angepasst werden muss.

Diesem Konzept folgend wird das Unternehmensvermögen als Geometrische Brown'sche Bewegung in kontinuierlicher Zeit modelliert, wobei Auszahlungen wie etwa bei Emanuel (1983, S. 1140) an die Investoren in das Unternehmensvermögen V stattfinden. Im Unterschied zum Grundmodell von Emanuel (1983) finden diese Auszahlungen zu diskreten Zeitpunkten statt, sodass die Integralform des Unternehmensvermögensprozesses nach Cont/Tankow (2004, S. 383)

$V_{t}=V_{0}+\int_{0}^{t}\left(\mu V_{s-}+D\right) \mathrm{ds}+\sigma \int_{0}^{t} V_{s-} \mathrm{dW}-\sum_{0<s<t ; s \in\left\{t_{1}, t_{2}, \ldots t_{l}\right\}} D$

aus drei Summanden besteht: (a) Der Diffusionskomponente, determiniert durch die Standardabweichung der Änderungen des Unternehmensvermögens $\sigma$ nebst W als standardnormalverteilte Zufallsvariable,(b) der Driftkomponente, determiniert durch die erwartete Änderung des Unternehmensvermögens $\mu$, und (c) die zu deterministischen Zeitpunkten stattfindenden Sprünge des Unternehmensvermögens. Letztere führen zu der cadlag (nur rechtsstetiger Prozess: $\mathrm{V}_{\mathrm{s}-}$ ) Eigenschaft des Prozesses und kommen durch Zinszahlungen auf das Fremdkapital L mit dem stetigen Satz $r_{L}$ zu Stande, welcher als konstant angenommen wird und unterhalb des risikolosen Zinssatzes liegt. Für einen Zeitraum $T_{i}-T_{i-1}$, der zwischen zwei Geschäftsjahren als konstant angenommen werden kann, lässt sich diese Zahlung am Geschäftsjahresende dann aus

$D=L \cdot\left(\exp \left(r_{L} T_{i}-r_{L} T_{i-1}\right)-1\right)$

und damit näherungsweise für jeden Zeitraum konstant ermitteln. Als weitere vereinfachende Annahme in Analogie zu Longstaff und Schwartz (1995) wird festgelegt, dass der Fremdkapitalbestand $\mathrm{L}$ als exogen gegebene Grösse angesehen wird.

Ferner wird angenommen, dass sämtliche Dividenden ebenso wie Auszahlungen für die Genussscheine stets sofort durch Einzahlungen derselben Klasse wieder ausgeglichen werden, sodass Dividendenzahlungen durch eine Kapitalerhöhung und Auszahlungen an die Genussscheininhaber durch Ausgabe neuer Genussscheine refinanziert werden. Diese Annahme ist zwar rigoros, verändert aber aufgrund der im Verhältnis zu D geringen Zahlungen das Modell nur unwesentlich.

Ferner wird, wie etwa bei allen strukturellen Modellen von Emanuel (1983) oder Realdon (2006, S. 4), der Bilanzgewinn oder Jahresüberschuss näherungsweise durch die Veränderung des Unternehmensvermögens (abzüglich der Zinsen auf das Fremdkapital am Geschäftsjahresende) bestimmt: 
$\Delta V_{i}=V_{i}-V_{i-.1}$

Diese Annahme ist ebenso diskussionswürdig, da insbesondere der Handelsbilanzbilanzgewinn aus dem Jahresüberschuss abgeleitet wird, welcher nur zum Teil den wahren Vermögenszuwachs der Gesellschaft widerspiegelt. ${ }^{13}$ Aufgrund der Abstraktion des Modells von Realität scheint sie aber ein akzeptabler Kompromiss.

Zudem müssen die üblichen Annahmen bzgl. der Marktusancen getroffen werden (siehe etwa Emanuel 1983, S. 1138, Merton 1977, S. 43 f. oder Trigeorgis 1996, S. 95 f.). Diese vorausgesetzt, kann ein Marktpreis des Risikos $\lambda$ abgeleitet werden, sodass sich für das nicht gehandelte Unternehmensvermögen eine Risikoprämie von $\lambda \sigma$ ergibt.

\section{Theoretischer Wert der Genussscheine}

\subsection{Risikoneutralisierter Preisprozess}

Mit den in 2.2 und 2.3 getroffenen Annahmen kann ein risikoneutralisiertes Mass bestimmt werden, unter dem eine Anlage in die gehandelten Vermögenswerte die erwartete Rendite abzüglich einer Risikoprämie erbringt. Als stochastischer Prozess dargestellt ergibt dies

$V_{T}=V_{0}+\int_{0}^{T}\left(\mu-\lambda \sigma-\frac{1}{2} \sigma^{2}\right) V_{s-} \mathrm{ds}+\sigma \int_{0}^{T} V_{s-} \mathrm{dW}_{s}-\sum_{0<s<T ; s \in\left\{t_{1}, t_{2}, \ldots t_{1}\right\}} D$

für den risikoneutralisierten Prozess. ${ }^{14}$ Die Behandlung der Zinszahlungen auf das Fremdkapital ist damit mit derjenigen vergleichbar, die bei der Bewertung von Optionen mit diskreten deterministischen Dividendenzahlungen (Wilmott 2006, S. 1040) angewendet wird, wenn man in Betracht zieht, dass die Zinszahlungen auf das Fremdkapital annahmegemäss dem Halter des Unternehmensvermögens V zufliessen.

\subsection{Wert des Genussscheins im Einperiodenfall}

Auf Basis der Annahmen lässt sich nun der Wert des Genussscheines im Bewertungstermin $\mathrm{t}=0$ vereinfacht bei Ausstehen eines Genussscheins im Volumen von $\mathrm{GK}_{0}$ bestimmen. Dabei steigt die Komplexität mit steigender Anzahl von Auszahlungsterminen an. Während für den trivialen Fall einer ausstehenden Zahlungen bei bekanntem Gewinn der Wert des Genussscheins sich als die risikolos diskontierte Abschlusszahlung errechnet, bestimmt sich der Wert im Falle eines noch unbekannten Gewinns über die Zahlungen in den oben skizierten Fällen. Formal ist dieser Fall gegeben, wenn die nominelle Restlaufzeit $\mathrm{T}_{1}$ positiv ist und gleichzeitig die nächste Zahlung weniger als ein Jahr vom Bewertungszeitpunkt $\Theta_{1}<1$ entfernt liegt. Die in diesem Fall ableitbare, geschlossene Lösung kann dann im weiteren Schritt dazu genutzt werden, die wesentlichen Werttreiber zu identifizieren.

Zur Wertermittlung des Genussscheins werden die möglichen Zahlungen in Abhängigkeit des Vermögens im nominellen Fälligkeitszeitpunkt (siehe Abbildung 2) benötigt. Es zeigen sich die drei möglichen Szenarien. Durch die Vollzahlung, bestehend aus Kupon,

13 Ferner ist bekanntlich der Bilanzgewinn wiederum durch die Gewinnverwendungsrechnung determiniert, welche die Ausschüttungsentscheidung beinhaltet.

14 Sämtliche Herleitungen sind auf der Verlagshomepage erhältlich. 
Nachzahlung und Nominalbetrag, ist der Zahlungsstrom nach oben begrenzt (Sektor 4). Demgegenüber findet sich im Falle einer Verlustbeteiligung eine quotale Abhängigkeit vom Vermögensendstand mit dem Faktor $\alpha$ (Sektor 2). Im Falle der Nachzahlung (Sektor 3) kommt der gesamte Vermögenszuwachs den Genussscheininhabern zu Gute. Ferner ist anzumerken, dass die Zahlung des Maximalbetrages die zusätzlichen unterjährigen Zinszahlungen beinhaltet. Dies ist bereits dann der Fall, wenn der Gewinn ausreicht, die Kuponzahlungen des Vorjahres zu bedienen. Dadurch entsteht ein Sprung in der Funktion, der mit der Tatsache erklärt werden kann, dass bei Erreichen der Gewinnschwelle, welche die maximale Nachzahlung und die Kuponzahlung ermöglicht, automatisch eine Ausschüttung des in diesem Beispiel halben zusätzlichen Kupons (Restlaufzeit 1 Jahr und 6 Monate) erfolgt.

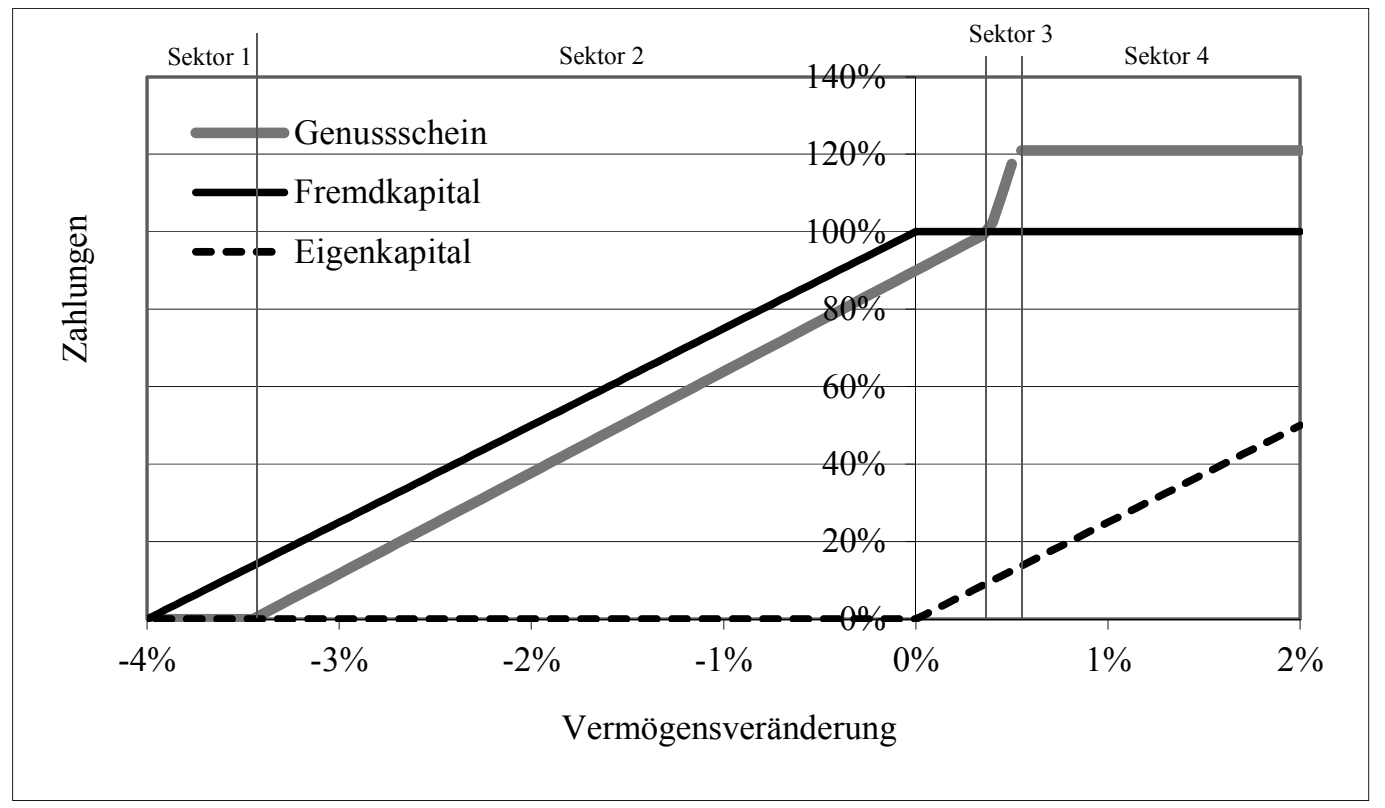

Abbildung 2: Auszahlungsprofil eines einjährigen Genussscheins in Verbindung zum Eigen- und Fremdkapital im Merton-Modell.

Daten: Vermögen 300 Mrd., 2 Mrd. Genussscheinnominalvolumen, Verlustbeteiligung $10 \%$, Kupon 6\%, Restlaufzeit 1,5 Jahre, Nachzahlung 2 Kupons, Genussrechtsanteil $17,4 \%$. Somit ergibt sich bei einer Vermögensänderung von Null eine Rückzahlung von $100 \%$ abzüglich einer Verlustbeteiligung von 10\%. Bei $+0,38 \%$ Vermögensänderung wird nur der Rückzahlungsbetrag ausgeschüttet. Die Maximalausschüttung besteht aus der Rückzahlung einer Nachzahlung von zwei Kupons wie auch dem regulären Kupon für 1,5 Jahre und beträgt $121 \%$. (Zahlungen im Black-Scholes-Merton-Modell für Fremd- und Eigenkapital rein fiktiv.)

Insgesamt lässt sich mit diesen Informationen der Wert eines einzelnen Genussscheins durch Erwartungswertbildung unter dem risikoneutralisierten Mass herleiten als

$P_{0,2 \text {. Fall }}=\mathrm{PV}_{A}^{\Theta 1}+\mathrm{PV}_{B}^{\Theta 1}+\mathrm{PV}_{C}^{\Theta 1}$ 
mit

$\mathrm{PV}_{A}^{\Theta 1}=e^{-r \Theta_{1}}\left(R+C\left(1+\Theta_{1}-T_{1}\right)+N_{0}\right)\left(1-\Phi\left(x_{1 a}\right)\right)$

$\mathrm{PV}_{B}^{\Theta 1}=\frac{V_{0}}{\mathrm{GK}_{1}} e^{-r \cdot \Theta_{1}+\mu_{\lambda} \cdot T_{1}}\left[\Phi\left(x_{1 b}\right)-\Phi\left(x_{2 b}\right)\right]-\left(\frac{V_{0}+D}{\mathrm{GK}_{0}}-\frac{\mathrm{VB}_{0}}{\alpha}\right) e^{-r \cdot \Theta_{1}}\left[\Phi\left(x_{1 a}\right)-\Phi\left(x_{2 a}\right)\right]+\operatorname{Re}^{-r \cdot \Theta_{1}}\left[\Phi\left(x_{1 a}\right)-\Phi\left(x_{2 a}\right)\right]$

$\mathrm{PV}_{C}^{\Theta 1}=\alpha \frac{V_{0}}{\mathrm{GK}_{1}} e^{-r \cdot \Theta_{1}+\mu_{\lambda} \cdot T_{1}}\left(\Phi\left(x_{2 b}\right)-\Phi\left(x_{3 b}\right)\right)-\alpha \frac{V_{0}+D}{\mathrm{GK}_{0}} e^{-r \cdot \Theta_{1}}\left(\Phi\left(x_{2 a}\right)-\Phi\left(x_{3 a}\right)\right)+\left(R-\frac{\mathrm{VB}_{0}}{\alpha}\right) e^{-r \cdot \Theta_{1}}\left(\Phi\left(x_{2 a}\right)-\Phi\left(x_{3 a}\right)\right)$

mit

$x_{1 a}=\frac{\ln \left(\frac{V_{0}+C \cdot \mathrm{GK}_{0}+N_{0} \cdot \mathrm{GK}_{0}-\mathrm{VB}_{0} \frac{\mathrm{GK}_{0}}{\alpha}+D}{V_{0}}\right)-\left(\mu-\lambda \sigma-\frac{1}{2} \sigma^{2}\right) T_{1}}{\sigma \sqrt{T_{1}}}$ und $x_{1 b}=x_{1 a}-\sigma \sqrt{T_{1}}$

$x_{2 a}=\frac{\ln \frac{V_{0}-\mathrm{VB}_{0} \frac{\mathrm{GK}_{0}}{\alpha}+D}{V_{0}}-\left(\mu-\lambda \sigma-\frac{1}{2} \sigma^{2}\right) T_{1}}{\sigma \sqrt{T_{1}}}$ und $x_{2 b}=x_{2 a}-\sigma \sqrt{T_{1}}$ und

$x_{3 a}=\frac{\ln \frac{L}{V_{0}}-\left(\mu-\lambda \sigma-\frac{1}{2} \sigma^{2}\right) T_{1}}{\sigma \sqrt{T_{1}}}$ und $x_{3 b}=x_{3 a}-\sigma \sqrt{T_{1}}$

In dieser Formel finden sich die drei Auszahlungssituationen, Gewinn ${ }^{15} \geq$ Zins- und Nachzahlungen $\left(\mathrm{PV}_{\mathrm{A}}\right)$, Gewinn < Zins- und Nachzahlungen $\left(\mathrm{PV}_{\mathrm{B}}\right)$ sowie Verlust $\left(\mathrm{PV}_{\mathrm{C}}\right)$ wieder. Dabei lässt sich der erste Summand $\mathrm{PV}_{\mathrm{A}}$ als Produkt aus risikoneutralisierter Wahrscheinlichkeit $\Phi()$, dass der Vermögenzuwachs ausreicht, um Kupons sämtlicher Nachzahlungen, Zinszahlungen, Verlustbeteiligungen auszugleichen, und dem maximalen Auszahlungsbetrag (siehe Abbildung 2, Sektor 4) interpretieren. Wichtig zu erwähnen ist, dass für eine vollständige Ausschüttung des Kupons in Höhe von $C\left(1+\Theta_{1}-T_{1}\right)$ nur ein Gewinn von $\mathrm{C}$ pro Genussschein nötig ist.

Der zweite Summand $\mathrm{PV}_{\mathrm{B}}$ kann über die risikoneutralisierten Wahrscheinlichkeiten interpretiert werden (Sektor 3). Dabei stellt der letzte Summand die erwartete Auszahlung des Rückzahlungsbetrages für den Fall „Gewinn < Zins- und Nachzahlungen“ dar. Die vorigen beiden Summanden repräsentieren die erwartete Zinszahlung, Gewinn abzüglich eines Ausgleichs der eventuell in 0 vorliegenden Verlustbeteiligung. Der erste Summand ist hier das erwartete Unternehmensvermögen pro Genussschein, der zweite das Ausgangsvermögen in 0. Die Unternehmensvermögensänderung muss dabei sowohl eine eventuelle Verlustbeteiligung der Genussscheininhaber also auch die der Eigenkapitalgeber ausgleichen, was durch den Divisor $\alpha$ erreicht wird.

Der Bereich der Verlustbeteiligung (Sektor 2) umfasst wiederum die erwartete Rückzahlung in diesem Szenario (letzter Summand) und den erwarteten anteiligen Verlust (erste beiden Summanden). Um diesen zu ermitteln, wird wiederum die Differenz des Unternehmensvermögens in Zeitpunkten 1 und 0 gebildet. Allerdings wird hier nur der den Genussscheininhabern zustehende Verlustanteil $(\alpha)$ berücksichtigt.

Für längere Zeiträume ist der Wert des Genussscheins unter Berücksichtigung mehrdimensionaler Verteilungen (vgl. ähnlich Geske 1977) und unter Zuhilfenahme rekursiver Formeln (Pfadabhängigkeit) darstellbar. Um das Integral zu lösen, kann hier auf numerische Verfahren (hier simulationsbasiertes Verfahren) zurückgegriffen werden. Bei mehre-

15 Siehe Fn. 10. 
ren Tests mit unterschiedlichen Volatilitäten zeigten sich auch für 10-jährige Genussscheine 25.000 Ziehungstupel als ausreichend konvergent.

\subsection{Interpretation und Werttreiber}

Zur Analyse der wesentlichen Werttreiber wird zunächst auf den Einperiodenfall zurückgegriffen, was aber grundsätzlich auf den Fall mehrere Perioden verallgemeinert werden kann. Auf den ersten Blick fällt die Vergleichbarkeit (etwa in Bezug auf gedeckelte Maximalauszahlung sowie fallende Auszahlung mit abnehmenden Unternehmensvermögen) mit der typische Auszahlungsfunktion eines Zerobonds wie im Merton-Modell auf, die auch im Mehrperiodenfall grundsätzlich erhalten bleibt. Aufgrund des dem Merton-Modell inhärenten Short-Put lässt sich damit grundsätzlich eine Abnahme des Genussscheinwerts mit steigender Volatilität postulieren. Dies ist dann besonders ausgeprägt, falls kein Nachzahlungsanspruch besteht. Ebenso nimmt der negative Wert des Short-Put bei langen Laufzeiten ab, wodurch bei gleichen Konditionen und flacher Zinskurve langlaufende Genussscheine, die mehrere Short-Puts beinhalten, meist niedrigere Werte aufweisen als kurzlaufende.

Dieses Bild verändert sich, falls im Bewertungszeitpunkt ein hoher Nachzahlungsanspruch unterstellt wird (siehe auch Abbildung Modell.2, Sektor 3), der aus früheren Perioden herrührt. Der Grund ist hier in der wertsteigernden Wirkung einer Art Outperformanceoption zu finden. Letztere entsteht durch die unterschiedliche Berücksichtigung von Verlusten (Anteil $\alpha$ ) und Nachzahlungen (Rudolph/Schäfer 2009, S. 379). Diese wird positiv von der Volatilität beeinflusst, sodass hier langlaufende Genussscheine mit mehreren Outperformanceoptionen unter Umständen auch höhere Werte als kurzlaufende Genussscheine annehmen können. ${ }^{16}$

Ist dagegen die Verlustbeteiligung hoch, kommt der Eigenkapitalcharakter zum Tragen. Sowohl die Outperformanceoption (bei niedrigem Nachzahlungsanspruch) als auch der Short-Put des Merton-Modells sind dann von untergeordneter Bedeutung, sodass der Genussscheinwert eher dem eines Long-Call ähnelt (Abbildung Modell.2, Sektor 2). Damit wirkt die Volatilität grundsätzlich wertsteigernd. Allerdings beinhaltet eine Laufzeitverlängerung nicht unbedingt eine Wertsteigerung, da bei längeren Laufzeiten ein Knock-Out durch Insolvenz zu früheren Zeitpunkten den Genussscheinwert vernichten kann.

\section{Empirische Untersuchung}

\subsection{Methoden des Modellvergleichs}

Die Bewertungsqualität des in den vorherigen Kapiteln entwickelten Modells wird nun mit den Marktpreisen von 130 Genussscheinen In-Sample und Out-of-Sample gegen die Ergebnisse von zwei Vergleichsmodellen überprüft. Der Vergleich mit Marktwerten entspricht dabei auch dem Vorgehen, das in zahlreichen verwandten Bereichen angewandt wird (siehe etwa Brown/Schaefer 1999 oder Jordan/Mansi 2003 bei Staatsanleihen, Wei/Guo 1997 bei ausfallrisikobehafteten Anleihen oder Ferreira et al. 1992 und Ramanlal et al. 1998 bei Vorzugsaktien).

16 Ein Beispiel für einen solchen Fall, bei dem die Preise der Genussscheine mit zunehmender Restlaufzeit steigen, sind die Genussscheine der Corealcredit am 26. Februar 2006. 
Einige Parameter, wie die Volatilität oder das Unternehmensvermögen, sind nicht direkt beobachtbar. Deswegen wird analog zu Bakshi et al. (2006) auf die Ermittlung impliziter Parameterwerte $\mathrm{V}_{0}$ und $\sigma$ aus den Genussscheinpreisen zurückgegriffen. Bei dieser Methode werden die Parameter so gewählt, dass die Differenz zwischen Modell- und Marktwerten der Anleihen an einem Handelstag die zu minimierende Variable ist. Im Einzelnen wird die maximale Abweichung der Preisdifferenzen tageweise für alle Genussscheine eines Emittenten minimiert:

$\min _{V_{0}, \sigma}\left(\max _{i}\left(P_{0, i}-P_{\text {Markt }, i}\right)\right)$

Dieses Vorgehen entspricht dabei der im Bereich der Anleihen üblichen Methode zum Vergleich von Modellen (vgl. Ericsson/Reneby 2005 oder Bakshi 1997, die Optionspreismodelle auch zunächst mit impliziten Volatilitäten kalibrieren). Grundsätzlich kann auf eine solche Methode zurückgegriffen werden, da, ebenso wie bei Anleihen, auch bei Genussscheinen mehrere Genussscheine eines Emittenten zum selben Zeitpunkt vorliegen, die unterschiedliche Laufzeiten aufweisen.

Auf Basis der so geschätzten Parameter wird dann in einem zweiten Schritt untersucht, wie gut die am Markt beobachteten Preise durch die Modellpreise erklärt werden können. Dabei wird sowohl ein In-Sample- als auch ein Out-of-Sample-Test eingesetzt (vgl. Ferreira et al. 1992, S. 58 ff.). Für den Out-of-Sample-Test werden die impliziten Parameter $\mathrm{V}_{0}$ und $\sigma$ als Durchschnitt der Werte des Vormonats (30 zeitlich vorgelagerte Handelstage) bestimmt, um sie dann zur Schätzung von $\mathrm{V}_{0}$ und $\sigma$ der jeweils folgenden Kurstage einzusetzen. Der Zeitraum von 30 Handelstagen wird dabei als Kompromisszeitraum gewählt. Einerseits birgt ein zu langer Zeitraum das Risiko von Änderungen der Einflussfaktoren durch neue wertbeeinflussende Ereignisse. Andererseits führt ein zu kurz gewählter Zeitraum dazu, dass nicht zu jedem Kurs im Schätzzeitraum genügend Daten für die Parameter vorhanden sind, weil nicht ausreichend viele umsatztragende Kurse vorhanden waren.

Zur Bestimmung des risikoneutralisierten Drift wird das Vorgehen von Ericsson und Reneby genutzt, das die risikoneutralisierte Rendite über den risikolosen Zins und die Zahlungen aus dem Unternehmensvermögen approximiert (Ericsson/Reneby 2004).

Da der Nutzen des alleinigen Tests eines Modells nur sehr eingeschränkt ist, wird ferner ein Vergleich an Hand zweier Referenzmodelle, nämlich

- des Merton-Modells als einfachem Vertreter der strukturellen Modelle (Merton 1974) und

- des Kanders-Modells als eigenkapitalorientiertes Genussscheinmodell (Kanders 1990, S. 91 und Schäcker 1997, S. 169 ff., der das Modell von Kanders für Spezialfälle implementiert)

vorgenommen.

Auf einen Vergleich mit dem in der Literatur vorgeschlagenen DCF-Modell wird verzichtet, da dieses Modell bislang nicht einmal erfolgreich bei Genussscheinen mit starkem Eigenkapitalcharakter angewendet werden konnte (Nowak/Hartmann 1995, S. 416. Beim Modell von Merton wird der Preis eines Genussscheins als

$P=\sum_{t=\Theta_{1}}^{\Theta_{K}-1} F\left(C, t, r_{t}, V, \sigma\right)+F\left(R+C\left(1+\Theta_{K}-T_{1}\right), \Theta_{K}, r_{K \Theta}, V, \sigma\right)$ 
bestimmt, wobei jeder Kupon, ebenso wie auch die Abschlusszahlung, als Zerobond im Sinne des Merton-Modells $\mathrm{F}\left(\right.$ ) bewertet wird und $\mathrm{r}_{\mathrm{t}}$ den laufzeitabhängigen Zins darstellt. Die beiden nicht direkt beobachtbaren Parameter Unternehmensvermögen und Volatilität werden wiederum implizit geschätzt.

Zur Verwendung des Modells von Kanders (1990) werden zusätzlich Gewinne, Erträge und Aufwendungen benötigt, die aus den HGB-Bilanzen übernommen werden. Der Parameter Volatilität der Erträge wird wiederum implizit geschätzt.

Um die Approximationsfähigkeit zu testen, wird zunächst die durchschnittliche Tschebyscheff-Norm angegeben. Für den Test der besseren Anpassbarkeit wird nach Langetieg/ Smoot (1989 S. 196 f.) ein Test auf signifikante Unterschiede in den Preisdifferenzen, eine Art t-Test, ebenso verwendet wie seine nichtparametrische Variante (siehe auch Bakshi et al. 2006). Die für Anleihemärkte konzipierten Tests sind auf Genussscheine übertragbar, da, wie oben beschrieben, die Situation zwischen Anleihen und Genussscheinen - was Fristigkeit etc. anbelangt - vergleichbar ist. Die Teststatistik basiert dabei im Wesentlichen auf einem Vergleich der Anpassungsgüte (Maximale Preisabweichung an einem Handelstag) zwischen den Modellen. Ein Modell ist dem anderen überlegen, wenn es an signifikant mehr Tagen eine geringere Preisabweichung aufweist.

\subsection{Datenauswahl}

Nachdem die EUWAX im Jahr 2000 ein eigenes Genussscheinsegment eröffnet hat, werden als Datenbasis sämtliche Genussscheine von Kreditinstituten verwendet, die zwischen Januar 2002 und Dezember 2009 an der EUWAX notiert wurden. Dadurch können 23 grosse Kreditinstitute in die Untersuchung aufgenommen werden. Auszuschliessen sind die zwei Genussscheine, welche keine fixe bzw. eine vom Referenzzinssatz abhängige Zinszahlung versprechen, sowie solche, die ein ordentliches Kündigungsrecht beinhalten. Die beiden ersten Ausschlusskriterien entspringen dabei direkt der Modellwahl, letzteres ist der Tatsache geschuldet, dass mindestens drei Genussscheine nötig sind, um die zwei Parameter $\mathrm{V}_{0}$ und $\sigma$ unter Einbeziehung eines Fehlerterms zu bestimmen.

Zudem besteht die Schwierigkeit, dass zahlreiche Fusionen der Emittenten stattfanden. Solange die Genussscheine sich auf den Gewinn der fusionierten Gesellschaft bezogen und keine Abweichungen in den Genussscheinbedingungen vorhanden waren, werden die Genussscheine vor der Fusion den einzelnen Emittenten und nach der Fusion dem fusionierten Kreditinstitut zugeordnet (vgl. zur Behandlung von Genussscheinen in diesem Fall Frantzen 1993, S. 273 ff. oder speziell für Banken Casper 2012). Abweichende Regeln der fusionierten Institute existierten allerdings bei drei Emittenten, der Corealcredit Bank (vormals Allgemeine Hypothekenbank Rheinboden AHBR), DZ-Bank und LBBW. Schlagend wurden diese Abweichungen allerdings nur bei der Corealcredit und der $L B B W$. Bei letzterer wird deswegen eine Schätzung mit und ohne Genussscheine der fusionierten Institute durchgeführt. Ferner wird bei der Corealcredit aufgrund der gravierenden Auswirkungen der Fusion neben der gemeinsamen Schätzung eine Schätzung separat für die Scheine der Rheinboden und der Allgemeinen Hypothekenbank durchgeführt. Einschliesslich der beiden Emittenten ergibt dies 23 Emittenten von 130 Genussscheinen. ${ }^{17}$

Da im Segment der Genussscheine nur ein Teil der Kurse auf Basis von Umsätzen festgestellt wurde und somit Kurse nur bedingt der aktuellen Marktlage entsprechen, werden

17 Weitere deskriptive Statistiken sind auf der Verlagshomepage erhältlich. 
grundsätzlich sämtliche Tax-, Brief und Geldkurse ausgeschlossen. Da dies bei den neun kleineren Emissionshäusern dazu führen würde, dass nur sehr wenige Kurstage zur Verfügung stünden, werden in Ausnahmefällen bei diesen auch Kurstage berücksichtigt, bei denen nur ein Kurs oder zwei Kurse vorhanden sind oder die bestehenden Kurse werden um einen weiterer nicht umsatztragender Kurs ergänzt. Da dies eventuell zu Verzerrungen führen kann, werden diese Emissionshäuser in der Analyse gesondert beachtet.

\subsection{Weitere Daten}

Zur Bestimmung der Insolvenzschwelle $\mathrm{L}$ wird vom Vermögen $\mathrm{V}_{0}$ sowohl das gesamte bilanzielle Eigenkapital zzgl. der Positionen nach $\mathbb{\$} 340 \mathrm{~g}$ HGB als auch das Genussrechtskapital abgezogen. Der Verlustbeteiligungsparameter $\alpha$ wird aus dem Wert des Genussrechtskapitals zur Summe aus Genussrechtskapital und Eigenkapital gemäss Genussscheinbedingungen bestimmt. Sämtliche Kapitalgrössen werden den für die Ausschüttungsbemessung relevanten HGB-Einzelabschlüssen entnommen.

Bei zwei Kreditinstituten, Corealcredit und IKB, werden die Genussscheine während des Betrachtungszeitraumes nicht mit Kuponzahlungen bedient. Die Angaben für die Verlustbeteiligung respektive für den Nachzahlungsanspruch werden dem Jahresabschluss entnommen. Bei den meisten Genussscheinen sind der Zahltag sowie die Zahlungshöhe der Genussscheine ex-ante bekannt. Bei 29 Genussscheinen ist er durch die Haupt-, Mitglieder- bzw. Generalversammlung determiniert. Hierfür wird der Tag und Monat der aktuell nächsten Versammlung als Annäherung für den künftigen Termin verwendet. Bei den sieben Genussscheinen, bei denen die Auszahlung vom kurzfristigen Zinssatz abhängt, werden die Terminzinssätze als Schätzer für die künftigen Sätze verwendet. Grundsätzlich werden die verwendeten Sätze für die risikolose Zinsstruktur von der Deutschen Bundesbank bezogen. Als Einlagezins wird der durchschnittliche Zinsaufwand in Relation zum Fremdkapital der einzelnen Institute genutzt.

\subsection{Studienergebnisse}

\subsubsection{Gesamtschau In- und Out-of-Sample}

Zunächst wird das Genussscheinmodell an die Marktdaten angepasst.

\begin{tabular}{|l|l|l|l|l|l|}
\hline & Minimum & Median & Mittelwert & Maximum & Standardabw. \\
\hline $\begin{array}{l}\text { Gewinn } \geq \text { Kupon- + } \\
\text { Nachzahlungen }\end{array}$ & $27,16 \%$ & $82,32 \%$ & $79,46 \%$ & $100,00 \%$ & $15,46 \%$ \\
\hline $\begin{array}{l}\text { Gewinn < Kupon- + } \\
\text { Nachzahlungen }\end{array}$ & $0,00 \%$ & $1,21 \%$ & $2,90 \%$ & $25,05 \%$ & $3,66 \%$ \\
\hline Verlust & $0,00 \%$ & $15,34 \%$ & $16,73 \%$ & $60,10 \%$ & $11,74 \%$ \\
\hline Insolvenz & $0,00 \%$ & $0,00 \%$ & $0,97 \%$ & $42,83 \%$ & $4,02 \%$ \\
\hline
\end{tabular}

Tabelle 2: Wahrscheinlichkeiten der verschiedenen Situationen

Wahrscheinlichkeiten der verschiedenen Auszahlungssituationen über alle Genussscheine aller Emittenten kalibriert auf die Marktpreise. 
Hierbei zeigt sich, dass der Fall (vgl. Tabelle 2), dass alle Kupon- und Nachzahlungen stets bedient werden, bei weitem der häufigste Fall ist. Der Insolvenzfall dagegen erweist sich bei der Kalibrierung des Modells als am wenigsten häufig. Einzig bei den Genussscheinen der Bayerischen Landesbank, von Correal und IKB spielt der Insolvenzfall eine Rolle. Gleiches gilt auch für den Fall des Verlustes und des nicht ausreichenden Gewinns. Gerade bei den Kursen aus dem Jahr 2008 und 2009 treten bei bestimmten Emittenten diese Fälle auf.

Bei der Überprüfung der Anpassungsfähigkeit In-Sample zeigt sich in vielen Datensätzen eine deutliche Tendenz zu Gunsten des einfachen Merton-Modells, wobei statistisch nicht die Fehlerdifferenz, wohl aber der nichtparametrische Zähltest signifikant ist (vgl. Tabelle 3). Das Merton-Modell ist in 18 von 25 Fällen statistisch signifikant überlegen. In 15 Fällen bei einem Konfidenzniveau von 1\%. Dagegen erweist sich das Kanders-Modell als grundsätzlich den beiden anderen Modellen unterlegen. Die schlechtere Anpassbarkeit scheint auf der Tatsache zu beruhen, dass bei diesem Modell mit der Volatilität nur ein anpassbarer Parameter zur Verfügung steht. Nur elf von 25 Fällen sind signifikant und nur fünf Fälle auf einem Signifikanzniveau von 1\%. 


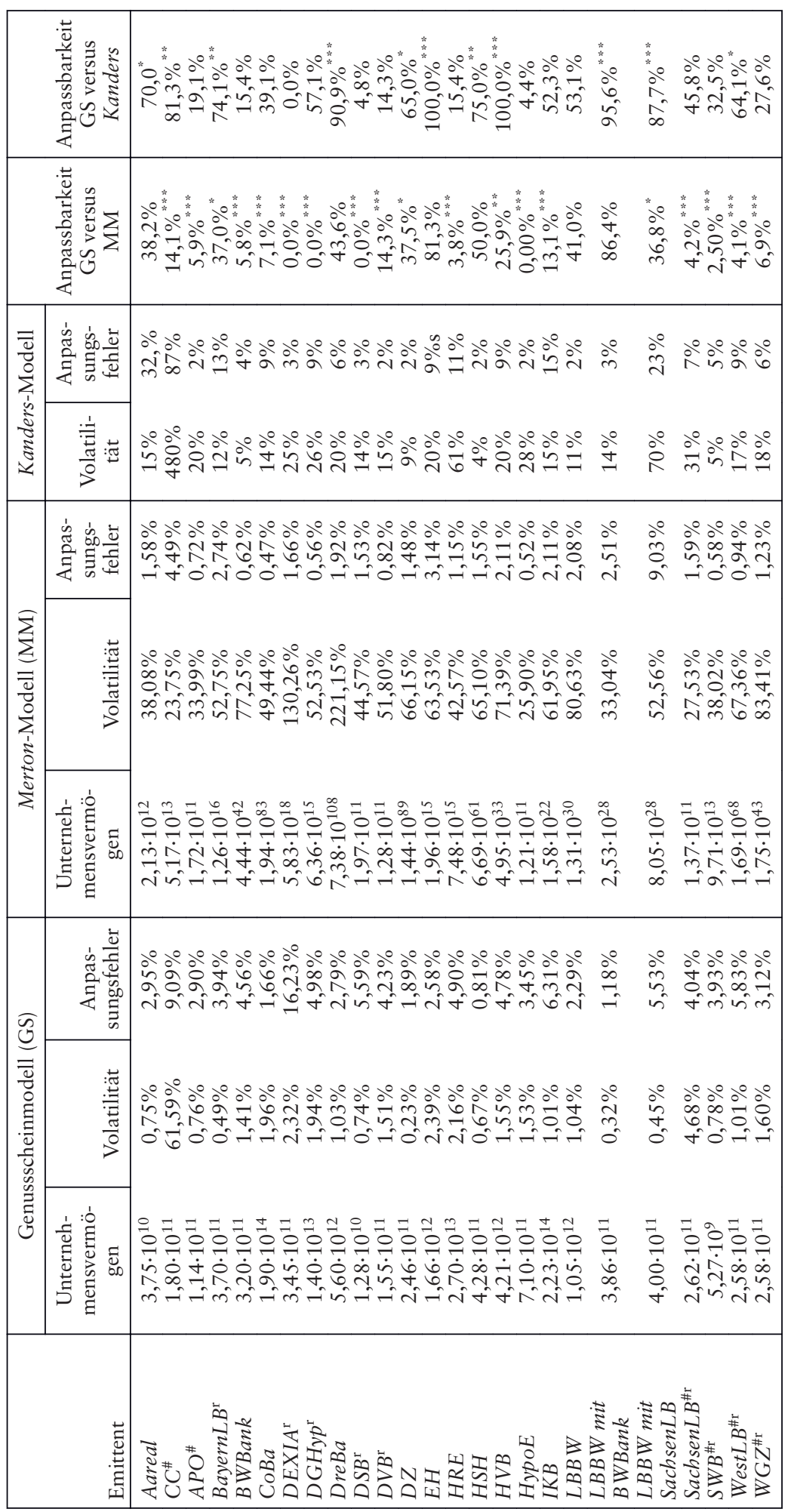

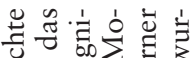

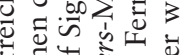

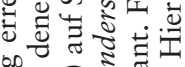

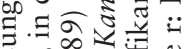

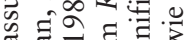

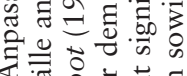

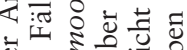

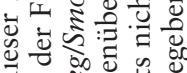

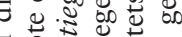

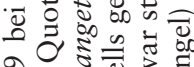

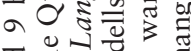

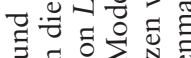

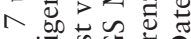
ब

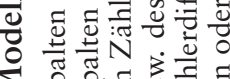

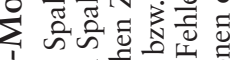
की

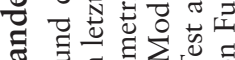

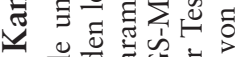
के क⿺辶寸

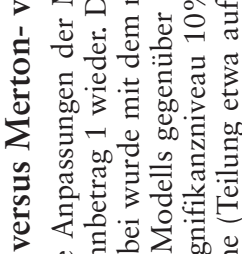

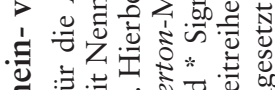

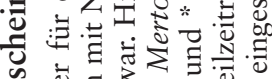

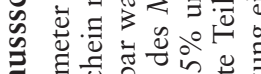

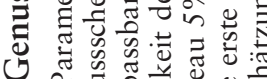
.

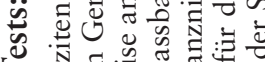
$H$ 정.

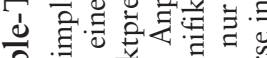

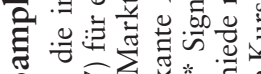
की

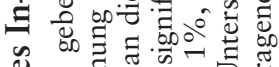

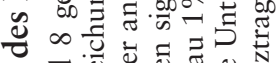

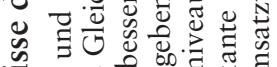
象

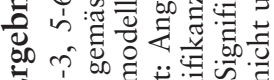
我 $\ddot{\sim} \overline{0}$ च

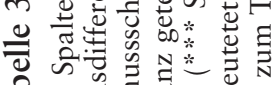

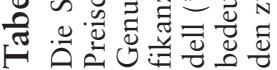


Bei der Analyse des Ergebnisses des Merton-Modells scheinen zwei Gründe für die Überlegenheit des Modells ursächlich zu sein: Erstens ist ein Grossteil der Genussscheine im Untersuchungszeitraum von Emittenten ausgegeben, die die Genussscheine stets bedient haben und deren Bilanzgewinn auch immer vollkommen ausreichend war, die Zahlungen zu bedienen. Damit trat der Eigenkapitalcharakter in den Hintergrund, sodass eine Messung mit einem Fremdkapitalmodell ebenso möglich war.

Zweitens stellt sich das Genussscheinmodell als eher parametersensibel heraus, sodass nur kleine Bewegungen der Inputparameter zu starken Schwankungen der Preise führen. Das lässt sich belegen, wenn die Schwankungen der Schätzparameter betrachtet werden. Beim Genussscheinmodell sind die Schwankungen durchgängig eher als niedrig zu bezeichnen. Beim Merton-Modell können sie dagegen exorbitante Grössen - man betrachte hier nur die durchschnittlichen Unternehmensvermögen, die in keiner ökonomisch sinnvollen Relation zur Bilanzsumme des Kreditinstituts (vgl. Tabelle 3) stehen - annehmen, was somit einen weiteren Beleg für die oben geäusserten Vermutungen darstellt.

Im Out-of-Sample-Test schneidet das Kanders-Modell aufgrund der partiell vorliegenden Überanpassung in allen Bereichen extrem schlecht ab. In etwa der Hälfte $(48 \%)$ der Fälle ist es signifikant unterlegen, in ca. $80 \%$ zeigt es schlechtere Anpassbarkeit. ${ }^{18}$ Ferner ist dem Merton-Modell nach Tabelle 4 im Out-of-Sample-Test keineswegs der Vorzug zu geben, sodass man sogar von einer gleichermassen guten Anpassbarkeit des Genussscheinmodells wie des Merton-Modells sprechen kann.

18 Alle Robustheitsanalysen sind auf der Verlagshomepage erhältlich. 


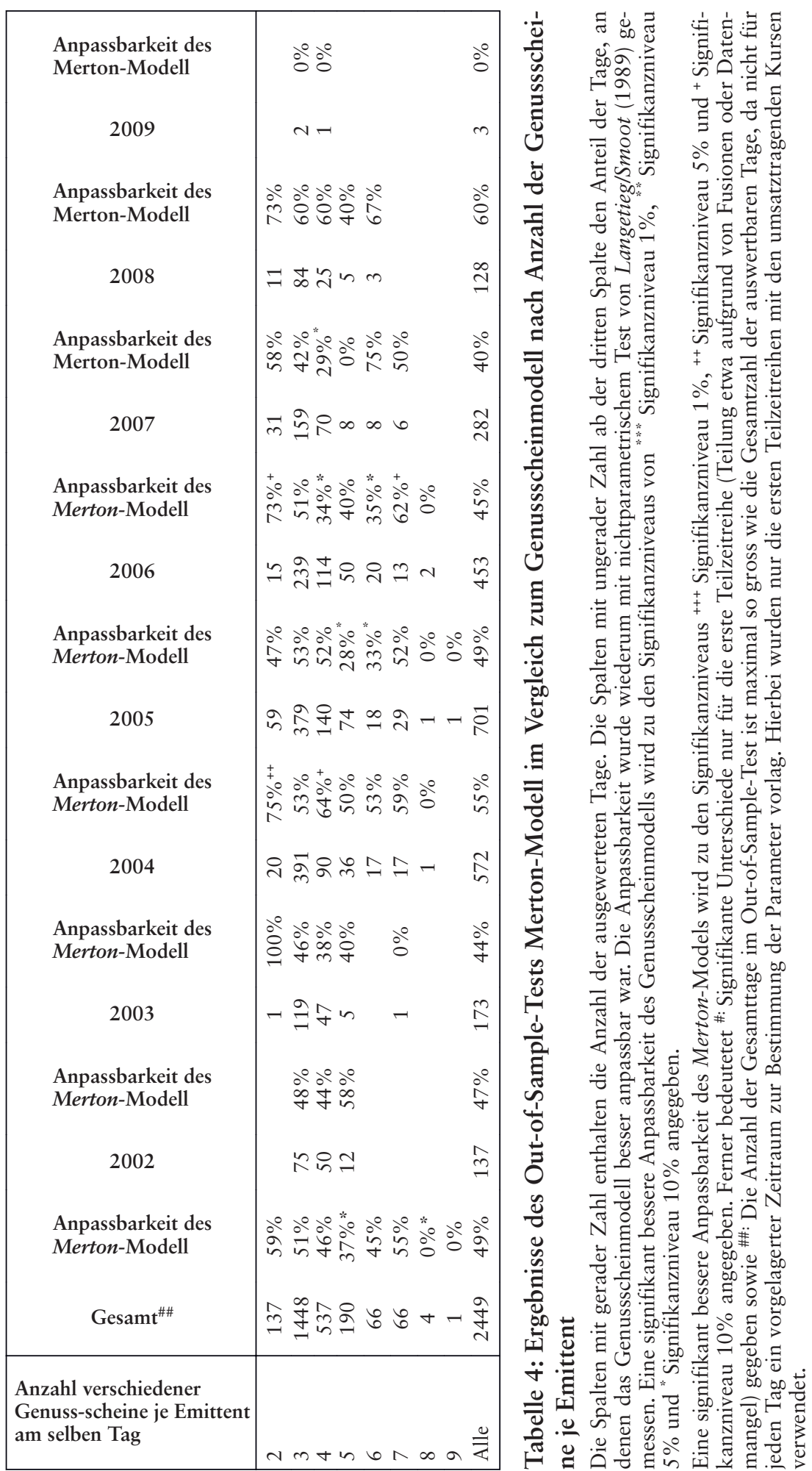


Um die Ergebnisse des Genussscheinmodells im Vergleich zum Merton-Modell zu analysieren, werden die Daten nach mehreren Kriterien aufgespalten. Dazu wird etwa untersucht, ob bei Kreditinstituten mit ausgesprochen hoher Volatilität des Unternehmensvermögens das Genussscheinmodell bessere Ergebnisse liefert, da bei diesen Instituten die Genussscheinbedingungen stärker zu Tage treten. Es zeigt sich, dass Emittenten wie die Bayerische Landesbank, Corealcredit, DVB oder IKB die höchste Volatilität des Unternehmensvermögens aufweisen, für die aber keine durchgehende bessere Anpassbarkeit des Genussscheinmodells festgestellt werden kann. Auch eine Analyse der Modelle im Zeitverlauf bringt keine Erkenntnisse, da auch in Zeiten der Finanzkrise das Merton-Modell und das Genussscheinmodel in etwa gleich abschnitten.

Zuletzt wird (siehe Tabelle 5) die Sensitivität der Modelle auf die Anzahl der je Emittent am selben Tag bewertbaren Genussscheine untersucht. Es zeigt sich, dass das Genussscheinmodell fast immer besser anpassbar ist, wenn mehrere Preisnotierungen verschiedener Genussscheine vom selben Emittenten gleichzeitig vorliegen. Dieser Effekt ist fast durchgängig beobachtbar und hat seine Ursache in folgendem Umstand: Im Merton-Modell sind nur gewisse Zinsstrukturkurven (Credit-Spread-Kurven) bei konstanten Zinssätzen erzeugbar (vgl. Pitts/Selby 1983). Wenn zahlreiche Genussscheinnotierungen an einem Tag vorliegen, kann die Lage der Preise zueinander, beeinflusst von Nachzahlungs- und Verlustbeteiligungsmöglichkeiten in Relation zur Laufzeit, verschiedene Formen annehmen. Diese aber können durch die vom Merton-Modell erzeugten einfachen CreditSpread-Kurven kaum noch abgebildet werden.

\section{Zusammenfassung}

Genussscheine wurden in der Vergangenheit häufig ähnlich wie nachrangige, festverzinsliche Wertpapieren eingestuft und bewertet. Dies war vor allem auf die sicheren Gewinne der Kreditinstitute zurückzuführen, die aufgrund der Anrechenbarkeit von Genussscheinkapital als Eigenkapital die wichtigsten Emittenten von Genussscheinen waren. Mit der Finanzkrise änderte sich diese Sichtweise, da trotz der Solvenz der Emittenten die im HGBAbschluss auftretenden Verluste zu einem Zahlungsausfall bei vielen Genussscheinen führten und damit den mezzaninen Charakter dieser Wertpapiere evident werden liessen. Bei der Bewertung von Genussscheinen wurde dieser Charakter jedoch bislang nur in Ansätzen berücksichtigt.

Deswegen wurde hier ein strukturelles Modell entwickelt, welches das Unternehmensvermögen als Geometrische Brown'sche Bewegung modelliert. Die Auszahlung an die Genussscheininhaber stellt dabei das Ergebnis der Veränderungen des Unternehmensvermögens dar und erfolgt aufgrund der im Wertpapierprospekt definierten Ausschüttungsregeln. Das Bewertungsergebnis für den Genussschein wird dabei im Wesentlichen durch drei Komponenten mit optionalem Charakter determiniert: Während die Begrenzung auf die vereinbarte Zins- und Tilgungszahlung im Sinne der Fremdkapitalbewertung als ShortPut interpretiert werden kann, stellt der vorrangige Nachzahlungsanspruch eine Art Outperformanceoption dar. Zuletzt führt die Knock-Out-Kaufoption dazu, dass bei Unterschreiten eines Schwellenwerts für die Insolvenz der Genussschein wertlos wird. Insgesamt überwiegt - je nach Nachzahlungsanspruch, Verlustbeteiligung oder Unternehmensvermögen im Verhältnis zur Insolvenzschwelle oder zu den vereinbarten Zins- und Tilgungsverpflichtungen - der Einfluss des Wertes der einen oder anderen Option. Betrachtet man das Zusammenspiel aller drei Optionen, sind bei Genussscheinen Situationen denkbar, welche 
auch am Markt beobachtet werden, in denen mit steigender Laufzeit der Wert der Genussscheine steigt, obwohl die risikolose Zinskurve und die vereinbarte Zahlungsstruktur eines Fremdkapitaltitels ein solches Verhalten eigentlich nicht zulässt. Theoretisch ist damit das Modell in der Lage, den mezzaninen Charakter der Genussscheine abzubilden.

Die Frage, ob das Modell die Marktpreise auch empirisch besser nachbildet, wurde an Hand einer Studie mit 130 Genussscheinen aus den Jahren 2002 bis 2009 analysiert. Anhand der umsatztragenden Preise von mehr als $90 \%$ aller börslich gehandelten Genussscheine wurde die Anpassungsgüte des Genussscheinmodells derjenigen des Merton-Modells und derjenigen des Kanders-Modells gegenübergestellt. Aufgrund der Schwierigkeiten der Beobachtbarkeit des Unternehmensvermögens und der Volatilität desselben wurden dabei diese Parameter an den über 3.300 Kurstagen implizit bei Vorliegen von zwei und mehr Genussscheinnotierungen bestimmt. In-Sample zeigte sich eine deutlich bessere Anpassbarkeit des einfachen Merton-Modells. Dies ist mit den Ergebnissen für amerikanische Vorzugsaktien vergleichbar, bei denen Ferreira et al. (1992) nachwiesen, dass das einfache Modell besser abschneidet. Out-of-Sample, d.h. bei Schätzung der impliziten Parameter in einer eigenen Schätzperiode, ging diese bessere Anpassbarkeit verloren. Insbesondere zeigte das Genussscheinmodell dann eine bessere Anpassbarkeit, wenn der mezzanine Charakter zum Tragen kam, was bei Vorliegen von mehr als drei umsatztragenden Kursen oft gegeben war. Das Kanders-Modell dagegen erwies sich beim Out-of-Sample-Test als weit unterlegen, da es mit der Volatilität der Erträge nur einen anpassbaren Parameter aufweist.

Insgesamt zeigt die empirische Untersuchung, dass in gewissen Situationen eine arbitragefreie Bewertung der Genussscheine mit Hilfe eines strukturellen Modells eine wesentliche Verbesserung gegenüber dem einfachen Merton-Modell und dem Kanders-Modell ergeben kann. Somit ist das Modell erstens geeignet, die von Industrieunternehmen begebenen und nicht börsennotierten mezzaninen Finanzinstrumente bewerten zu können. Zweitens kann aus dem vorliegenden Ergebnis gefolgert werden, dass auf dem Genussscheinmarkt mit seinen hochspeziellen Anlageinstrumenten die Marktteilnehmer die spezifischen Genussscheincharakteristika mit in die Bepreisung einbeziehen.

Offen bleibt, inwieweit das Modell auch für Nichtstandardfälle, wie etwa Genussscheine, deren Zahlungen von Dividenden, Gesamt- oder Umsatzrenditen abhängen, erweiterbar ist. Auch eine Modellanpassung an weitere Emissionspreise nicht börsengehandelter mezzaniner Finanzinstrumente erscheint hier ebenso vielversprechend.

\section{Literaturverzeichnis}

Bakshi, G./ Cao, C./ Chen, Z. (1997): Empirical performance of alternative option pricing models, in: Journal of Finance, Jg. 52, S. 2003-2049.

Bakshi, G./ Madan, D. B./ Zhang, F. X. (2006): Recovery risk in defaultable debt models: Empirical comparisons and implied recovery rates. Working Paper 2006.

Brokamp, J./ Ernst, D./ Hollasch, K./ Lehmann, G./ Weigel, K. (2008): Mezzanine-Finanzierungen, München.

Brown, R./ Schaefer, S. (1994): The term structure of real interest rates and the Cox, Ingersoll, and Ross model, in: Journal of Financial Economics, Jg. 35, S. 3-42.

Casper, M. (2012): Genussscheine von Banken nach einer Konzernierung des Emittenten, in: Zeitschrift für Wirtschaftsrecht, Jg. 33, S. 497-503.

Cont, R./ Tankow, P. (2004): Financial modelling with jump processes, Boca Raton et al. 
DGAP (2010a): COREALCREDIT BANK AG erwirtschaftet 2009 positives Ergebnis

Ad Hoc Meldung am 15.4.2010 um 13:44.

DGAP (2010b): IKB Deutsche Industriebank AG: Ergebnisse des Geschäftsjahres 2009/10, Ad Hoc Meldung am 1.7.2010 um 08:00.

Eberhartinger, E. (1996): Bilanzierung und Besteuerung von Genußrechten, Wien.

Emanuel, D (1983): A theoretical model for valuing preferred stocks, in: Journal of Finance Jg. 38, S. 1133-55.

Ericsson, J./ Reneby, J. (2004): A note on contingent claims pricing with non-traded assets, in: Finance Letters, Jg. 2, H. 3.

Ericsson, J./ Reneby, J. (2005): Estimating structural bond pricing models, in: Journal of Business, Jg. 78, S. 707-35.

Ernst, T. (1963): Der Genußschein im deutschen und schweizerischen Aktienrecht, Zürich.

Ferreira, E. J./ Spivey, M. F./ Edwards, C. E. (1992): Pricing new-issue and seasoned preferred stocks: A comparison of valuation models, in: Financial Management, Jg. 21, S. 52-62.

Frantzen, C. (1993): Genußscheine: zugleich eine Analyse der Genußscheinbedingungen deutscher Unternehmen, Köln et al.

Gehling, C (1992): Obligationsähnliche Genußrechte. Zeitschrift für Wirtschafts- und Bankrecht Wertpapiermitteilungen, Jg. 46, S. 1093-1100.

Geske, R. (1977): The valuation of corporate liabilities as compound options, in: Journal of Financial and Quantitative Analysis, Jg. 12, S. 541-552.

Götte, R. (2007): Aktienanleihen, Discount-Zertifikate, Fonds, Genussscheine. 2. Auflage, Marburg.

Hirte, H. (2001): Genussscheine, in: Gerke W./ Steiner, M. (Hrsg): Handwörterbuch des Bank- und Finanzwesens, 3. Auflage, Stuttgart, Sp 967-74.

Jordan, J./ Mansi S. (2003): Term structure estimation from on the run treasuries, in: Journal of Banking and Finance, Jg. 27, S. 1487-1509.

Kanders, G. (1990): Bewertung von Genußscheinen. Berlin.

Kühn, U. (2008): Genussscheine: Chance für Mutige, in: Focus Money Jg. 53, H. 3.

Langetieg T./ Smoot S. (1989): Estimation of the term structure of interest rates, in: Research in Financial Services, Jg. 1, S. 181-222.

Longstaff F. A./ Schwartz, E. S. (1995): A simple approach to valuing risky fixed and floating rate debt, in: Journal of Finance, Jg. 50, S. 789-819.

Lühn, M. (2006): Bilanzierung und Besteuerung von Genussrechten, Wiesbaden.

Lühn, M. (2012): Genussrechte als Finanzierungselement mittelständischer Unternehmen, in: Plate, G. (Hrsg.): Forschung für die Wirtschaft, Göttingen, S. 73-90.

Lühn, M. (2013): Genussrechte- Grundlagen, Einsatzmöglichkeiten, Bilanzierung und Besteuerung, Wiesbaden.

Merton, R. C. (1974): On the pricing of corporate debt: The risk structure of interest rates, in: Journal of Finance, Jg. 41, S. 449-470.

Merton, R. C. (1977): On the Pricing of Contingent Claims and the Modigliani Miller Theorem, in: Journal of Financial Economics, Jg. 5, S. 241-249.

Nowak T./ Hartmann B. (1995): Bewertung von Genußscheinen, in: Die Bank, Jg. 19, S. 412-417.

Pitts, C./ Selby, M. (1983): The pricing of corporate debt: A further note, in: Journal of Finance, Jg. 38, S. 1311-1313. 
Ramanlal, P./ Mann, S. V./ Moore, W. T. (1998): Convertible preferred stock valuation: Tests of alternative models, in: Review of Quantitative Finance and Accounting, Jg. 10, S. 303-319.

Realdon, M. (2006): Revisiting cumulative preferred stock valuation, in: Finance Research Letters, Jg. 3, S. 2-13.

Rottwilm, C. (2010): Der Markt für Genusscheine trocknet aus, in: manager magazin 9/2010 am 16.9.2010.

Rudolph, B./ Schäfer K. (2009): Derivative Finanzinstrumente, 2. Auflage, Berlin et al.

Schäcker, H.-A. (1997): Börsenpreise von Genußscheinen - Empirische Überprüfung eines theoretischen Bewertungsansatzes, Berlin.

Steinbach, M. (1999): Der standardisierte börsennotierte Genußschein, Wiesbaden.

Trigeorgis, L. (1996): Real Options, Cambridge.

Wei D. G./ Guo, D. (1997): Pricing risky debt: an empirical comparison of Longstaff and Schwartz and Merton models, in: Journal of Fixed Income, Jg. 7, H. 2, S. 8-28.

Westphal, H. (1995): Gestaltung und Bewertung von Genußscheinkapital - Chancen und Risiken aus Emittenten- und Anlegersicht, Wiesbaden.

Wilmott, P. (2006): Paul Wilmott on Quantitative Finance. 2. Auflage, Chichester et al.

Henning Fock, Lehrstuhl für Finanz- und Informationsmanagement, Mathematisch-Naturwissenschaftlich Technische Fakultät der Universität Augsburg.

Anschrift: Universität Augsburg, Universitätsstr. 2, 86159 Augsburg, Deutschland

Andreas Rathgeber, Professur für Finanz- und Informationsmanagement, MathematischNaturwissenschaftlich Technische Fakultät der Universität Augsburg (Korrespondierender Autor: andreas.rathgeber@mrm.uni-augsburg.de).

Anschrift: Universität Augsburg, Universitätsstr. 2, 86159 Augsburg, Deutschland 